\title{
Measurements, system response, and calibration of the SLAC T-510 Experiment
}

\section{Stephanie A. Wissel ${ }^{* a}{ }^{a}$ K. Bechtol, ${ }^{b}$ K. Belov, ${ }^{c, a}$ K. Borch, ${ }^{a}$ P. Chen, ${ }^{d}$ J. Clem, ${ }^{e}$ P. W. Gorham,${ }^{f}$ C. Hast, ${ }^{g}$ T. Huege, ${ }^{h}$ R. Hyneman,,${ }^{i, a}$ K. Jobe,${ }^{g}$ K. Kuwatani,${ }^{a}$ J. Lam, ${ }^{a}$ T. Liu, ${ }^{f}$ K. Mulrey, ${ }^{e}$ J. Nam, ${ }^{d}$ C. Naudet ${ }^{c}$ R. Nichol, ${ }^{j}$ B. F. Rauch, ${ }^{k}$ A. Romero-Wolf, ${ }^{c}$

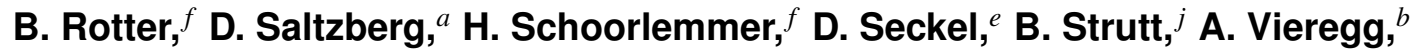 C. Williams, ${ }^{l}$ and A. Zilles ${ }^{m}$}

${ }^{a}$ Dept. of Physics and Astro., Univ. of California, Los Angeles, Los Angeles, CA 90095, USA.

${ }^{b}$ Dept. of Physics, University of Chicago, Chicago, IL, USA.

${ }^{c}$ Jet Propulsion Laboratory, Pasadena, CA 91109, USA.

${ }^{d}$ Dept. of Physics, Grad. Inst. of Astrophys., \& Leung Center for Cosmology and Particle Astrophysics, National Taiwan University, Taipei, Taiwan.

e Dept. of Physics, Univ. of Delaware, Newark, DE 19716, USA.

${ }^{f}$ Dept. of Physics and Astronomy, Univ. of Hawaii, Manoa, HI 96822, USA

g SLAC National Accelerator Laboratory, Menlo Park, CA, 94025, USA.

${ }^{h}$ Karlsruher Inst. für Tech., Inst. für Kernphysik, 76021 Karlsruhe, Germany.

${ }^{i}$ Physics Dept., College of William \& Mary, Williamsburg VA 23187, USA.

${ }^{j}$ Dept. of Physics and Astronomy, University College London, London, United Kingdom.

${ }^{k}$ Dept. of Physics, Washington Univ. in St. Louis, St. Louis, MO 63130, USA.

${ }^{l}$ Dept. of Physics, Stanford University, Stanford, CA, 94305, USA.

${ }^{m}$ Karlsruher Inst. für Tech., Inst. für Experimentelle Kernphysik, 76128 Karlsruhe, Germany Email: swisselephysics.ucla.edu

The SLAC T-510 experiment provides the first beam-test of radio-frequency radiation from a charged particle cascade in the presence of a magnetic field (up to $970 \mathrm{G}$ ), a model system for radio-frequency emission from a cosmic-ray air shower. The primary purpose of this experiment is to provide a suite of controlled laboratory tests to compare to simulations based on particlelevel models of RF emission, making the calibrations of critical importance. We present system calibrations and analysis of the experiment from end to end. Measurements of the beam charge and two-dimensional magnetic field map are fed directly into the simulations using two different formalisms: ZHS and Endpoints. Simulated electric fields are forward-folded with the system response, allowing for direct comparisons of spectra and waveforms with the simulations.

The 34th International Cosmic Ray Conference,

30 July- 6 August, 2015

The Hague, The Netherlands

\footnotetext{
*Speaker.
} 


\section{Overview}

The SLAC T-510 experiment is the first beam experiment of the radio emission from particle showers in a strong magnetic field. The experiment was designed as a model system of the radio emission from ultra-high energy cosmic-ray air showers. Its primary goal is to provide a suite of controlled laboratory measurements that can be compared to models of radio emission, such as ZHAireS [1] and CoREAS [2].

The T-510 experiment was carried out in End Station A at the SLAC National Accelerator Laboratory. An electron beam of energy $4.35 \mathrm{GeV}$ or $4.55 \mathrm{GeV}$ impacted a high-density polyethylene (HDPE) target after traversing two radiation lengths of lead. The density of the HDPE (index of refraction, $n=1.53$ ) compressed the shower relative to an air shower such that the majority of the shower was contained within the $4 m \times 0.96 m \times 0.60 m$ target. Magnetic field coils underneath the target generated a controllable magnetic field of up to 970 Gauss, perpendicular to the beam direction.

Two main mechanisms can be used to describe the radio frequency (RF) emission in the SLAC T-510 geometry. Radiation due to charge excess built up in the shower, also known as the Askaryan effect [3, 4], is radially polarized, and therefore, appears vertically polarized when the antenna array is centered on the beam in this experiment. This radiation is not expected to be dependent on the strength of the magnetic field. Radio emission is also produced from the transverse current generated by applying a vertical magnetic field. In the T-510 geometry, this radiation is expected to be the dominant component of the horizontally polarized emission.

A vertically-oriented array of antennas recorded the electric field at various locations downstream of the beam and target. We moved the array both vertically and horizontally away from the beam via an overhead crane in order to map out the angular dependence of the radio emission. The primary data were taken with quad-ridge horn antennas, originally designed for the ANITA experiment [5], covering frequencies between 200 and $1200 \mathrm{MHz}$. The antennas are broadband and have two orthogonal linear polarizations. The gain is approximately $10 \mathrm{~dB}$. The induced voltages on the antennas were recorded on $2.0 \mathrm{GHz}$ oscilloscopes after passing through $15.24 \mathrm{~m}$ of LMR 240 coaxial cable and a $1250 \mathrm{MHz}$ low pass filter to avoid aliasing. During the primary data run, the antenna array was placed $13.47 \mathrm{~m}$ from the entrance of the beam on the target. For an overview of the experiment and its results, see Belov et al. in these proceedings [6].

The models of radio-frequency (RF) emission used in CoREAS and ZHAireS are Endpoints and ZHS, respectively. Combined with the GEANT 4.10 toolkit, they provide the predictions for the radio emission in the SLAC T-510 experiemnt. are discussed in more detail in [7]. We describe here the experimental data and system calibration used for such comparisons.For a discussion of the simulations developed for this experiment, see Zilles et al. in these proceedings [7].

\section{Magnetic Field}

Fifteen water cooled solenoids were used to produce a vertical magnetic field. The magnetic field coils were staggered on top of each other in order to make the field more uniform. The bottom row had eight coils placed side-by-side. The top row included seven coils that covered the full length of the target. We controlled the strength of the magnetic field by supplying up 


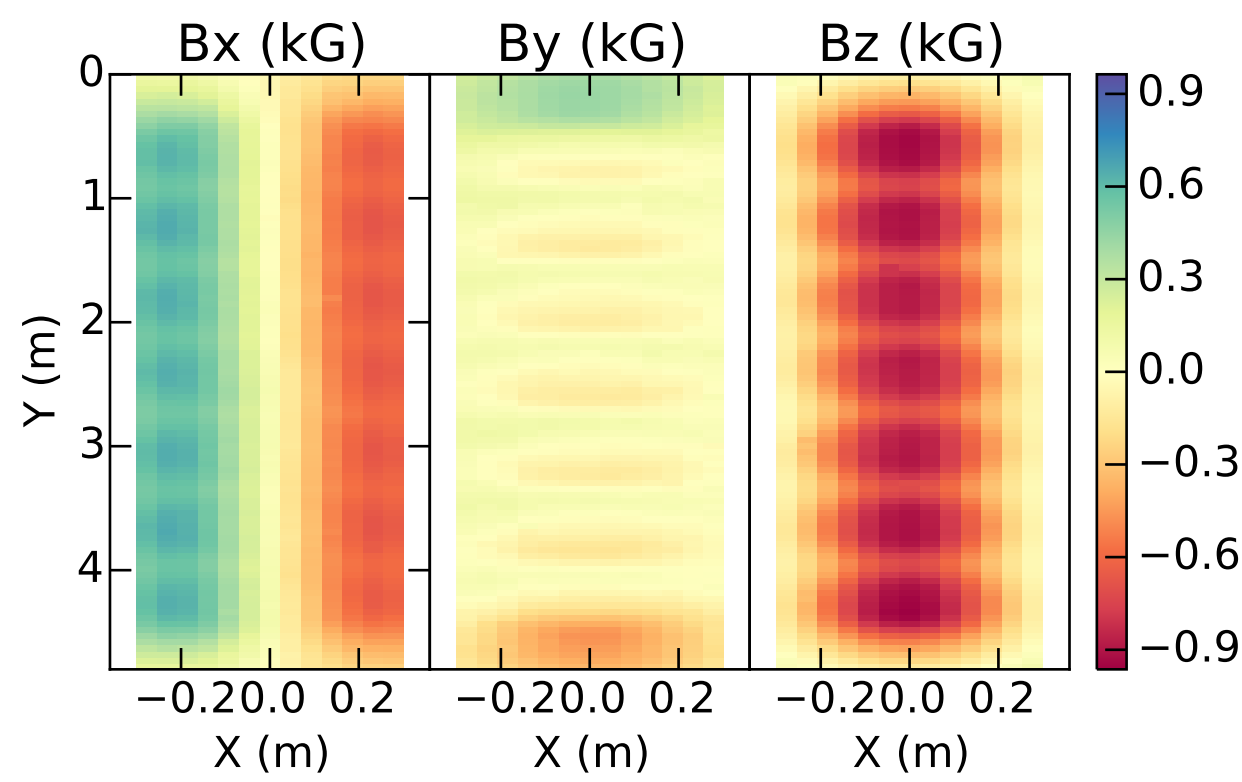

Figure 1: The measured magnetic field at the beam height along the axes transverse $(x)$, longitudinal $(y)$, and vertical $(z)$ to the beam

to 2530 Amperes direct current to three sets of five coils. Together the coils generated a vertical magnetic field of up to \pm 970 Gauss. The magnetic field generated by these coils was strong enough to bring the radiation intensity from the magnetic effect to the same order of magnitude as from the Askaryan effect.

The magnetic field map at maximum current is shown in Fig. 1, measured at the beam height, $21 \mathrm{~cm}$ above the coils with $5 \mathrm{~cm} \times 5 \mathrm{~cm}$ grid spacing and $3.64 \mathrm{G}$ precision. There is a $5.73 \%$ uncertainty on the magnetic field measurements arising largely from the average percent error of the Hall probe used. Because the vertical $\left(B_{z}\right)$ field falls off near the edges of the coils, we placed the edge of the target in the middle of the first coil. Along the beam position, the average magnetic field in the vertical direction is $-845 \mathrm{G}$, and the root-mean-squared variation is $72 \mathrm{G}$. In our simulations of the T-510 experiment, we included the measured, three-dimensional magnetic field [7].

\section{Beam Monitoring}

An integrating charge transformer (ICT) placed between the beam pipe and the target monitored the total charge in each bunch. A high-frequency S-band (2-4 GHz) horn antenna also recorded the transition radiation as the beam exited the pipe and provided a global trigger for the system as well as the shot-to-shot relative calibration of the beam charge. The signals from both monitoring systems were recorded on a $3.0 \mathrm{GHz}$ oscilloscope.

The intensity of the transition radiation emitted as the beam exited the beam pipe scales with the total charge. This is shown in Fig. 2(b) where the peak amplitude recorded in the S-band horn is compared to the bunch charge. These data were recorded by varying the diameter of the collimator on the beam, thereby reducing the total charge exiting the pipe. During the main data 


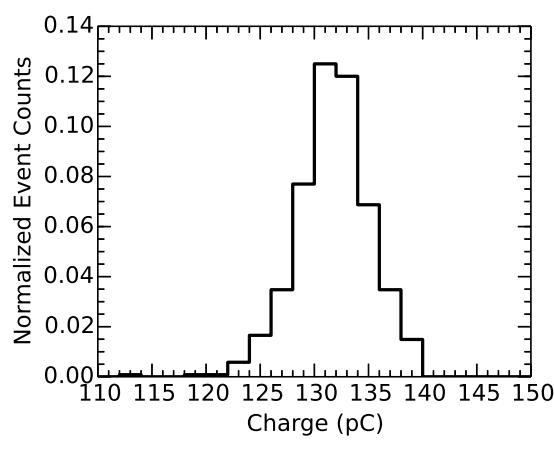

(a)

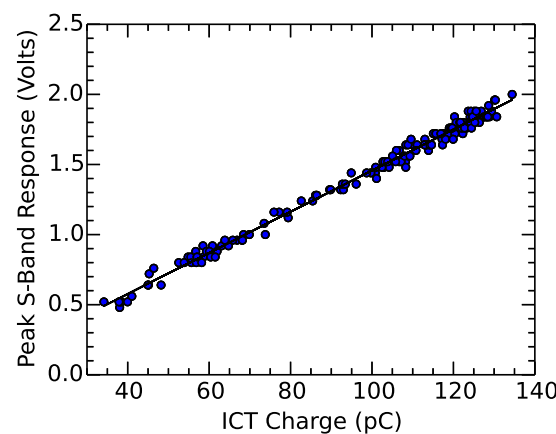

(b)

Figure 2: (a) The typical shot-to-shot beam charge and (b) its relationship to the induced voltage on the S-band horn.

runs, the collimators made as wide as possible $(10 \mathrm{~cm}$ diameter $)$, thereby reducing the variation in shot-to-shot charge.

Charge induced in the ICT gets stored on capacitors that are then read out slowly through a readout transformer. The ICT was calibrated with a fast pulse generator that injected charge between 6 and $450 \mathrm{pC}$ on timescales varying between 2 and $4 \mathrm{~ns}$. It is accurate to within 3\% for bunch charges greater than $100 \mathrm{pC}$. The charge distribution measured by the ICT for a typical run, shown in Fig. 2(a), has a mean of $131 \mathrm{pC}$ and a standard deviation of $3.3 \mathrm{pC}$. The shot-to-shot beam charge during the T-510 experiment remained constant to within $3 \%$.

\section{Data Cleaning}

In our antennas, the vertical signal will leak into the horizontally polarized channel at about the $25 \%$ level in voltage. We eliminate this leakage by construction by analyzing the difference between field-up, $V_{B_{+}}(t)$, and field-down, $V_{B_{-}}(t)$, data, namely $V(t)=\frac{1}{2}\left(V_{B_{+}}(t)-V_{B_{-}}(t)\right)$. Each waveform is scaled by a nominal shot beam charge to $131 \mathrm{pC}$, estimated by the amplitude of the transition radiation recorded by the S-band horn. Each waveform is also zero-meaned to remove any DC offset in the data. Two horizontally polarized waveforms with the cross-polarization contamination removed are shown in Fig. 3.

Reflections off of the bottom of the target interfered with the main pulse, which is apparent $1.5 \mathrm{~ns}$ and $6 \mathrm{~ns}$ after the initial pulse in Fig. 3. One reflection arrived at a given antenna position about $1 \mathrm{~ns}$ after the main pulse with inverted polarity, and largely constructively interfered with it. A second reflection bounced off the top of the target and again off the bottom surface, arriving 4-6 ns after the first pulse. For the spectral comparisons that follow, we window the data $\pm 10 \mathrm{~ns}$ around the peak, which retains the reflections in the spectra, but removes extraneous noise.

\section{Antenna and System Response}

Simulation results from the ZHS and Endpoints formalisms are convolved with the measured system response of the cables and filters for each channel and the effective height of the antennas so that the measured voltages can be compared directly with the models. The time-domain voltage, 
$V(t)$, at the antennas is a convolution (Eqn. 5.1) of the antenna impulse response, $h_{e f f}(t)$, and the system impulse response due to the filters and loss of the cables, $h_{s y s}(t)$, with the simulated electric field, $E(t)$. This is equivalent to the multiplication of the effective height, $H_{e f f}(f)$, with the system response, $H_{s y s}(f)$, with the electric field in the frequency domain (Eqn. 5.2) [8].

$$
\begin{array}{r}
V(t)=h_{e f f}(t) \circ h_{s y s}(t) \circ E(t) \\
V(f)=H_{e f f}(f) H_{s y s}(f) E(f)
\end{array}
$$

Because the radiation is expected to peak at the Cherenkov angle of $29.8^{\circ}$, the horn antennas were tilted $19.6^{\circ}$ down towards the target. Over the entire vertical scan the angle between the boresight of the antennas and the peak of the expected radiation varied by $\pm 20^{\circ}$. Fig. 4(a) shows the effective height of the antennas as a function of frequency for angles off boresight covered in the vertical scan. The relative gain, and therefore, the effective height, of the antennas varies by $3.7 \mathrm{~dB}$ for the vertical polarization and by $2 \mathrm{~dB}$ for the horizontal polarization. The system response is dominated by the $1250 \mathrm{MHz}$ low pass filter, which drives the sharp increase in loss shown in Fig. 4(b).

\section{Data and Model Comparisons}

Fig. 5 shows power spectral densities measured at several positions (1.30 m, $6.52 \mathrm{~m}, 11.29 \mathrm{~m})$ above the beam height and $13.47 \mathrm{~m}$ from the point where the beam enters the target. The spectra are compared with simulations following the ZHS and Endpoints formalisms [7] that are convolved with the effective height and system response shown in Fig. 4. All of the spectra are plotted on the same scale to illustrate the increase in Fourier amplitudes near the Cherenkov angle.

The right-hand side of Fig. 5 shows the vertically polarized emission, which remains constant relative to the applied magnetic field strength. The models compare well with the measurements. The integrated power in the $300-1200 \mathrm{MHz}$ band is strongest in the center, and is an order of magnitude lower at the edges of the vertical scan. Similarly, the horizontally polarized emission, shown on the left of Fig. 5, grows by an order of magnitude towards the center of the vertical scan.

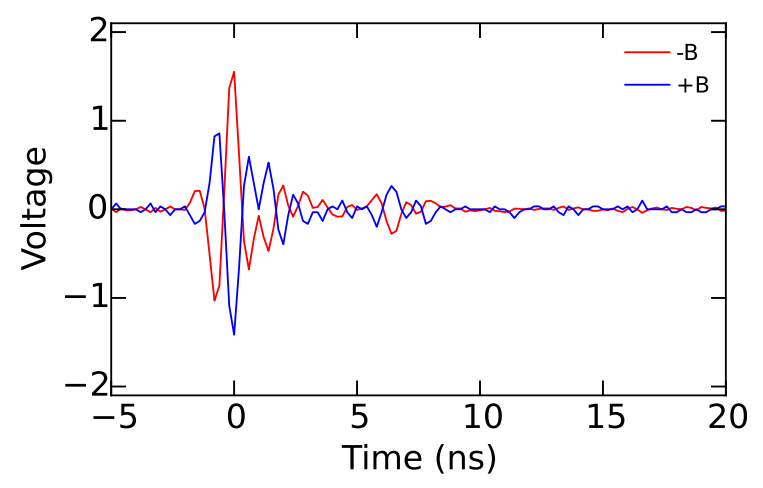

Figure 3: Measured horizontally polarized waveforms corrected for cross-polarization leakage. The positive B-field is shown in blue; the negative, in red. 


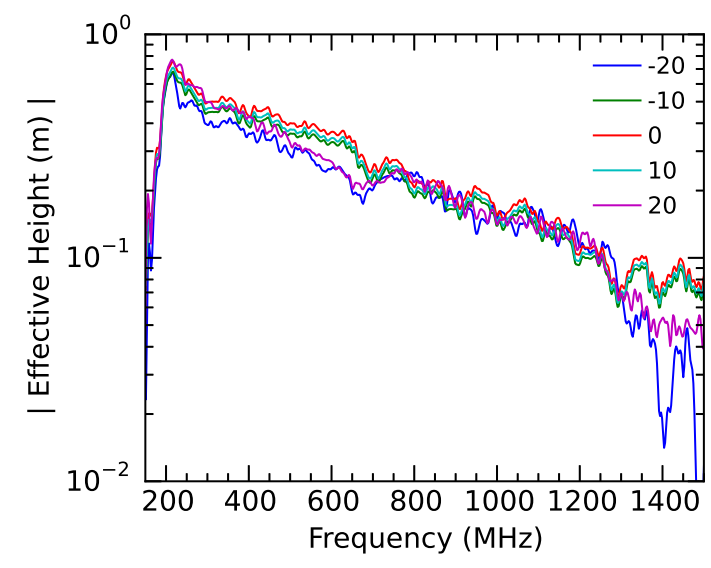

(a)

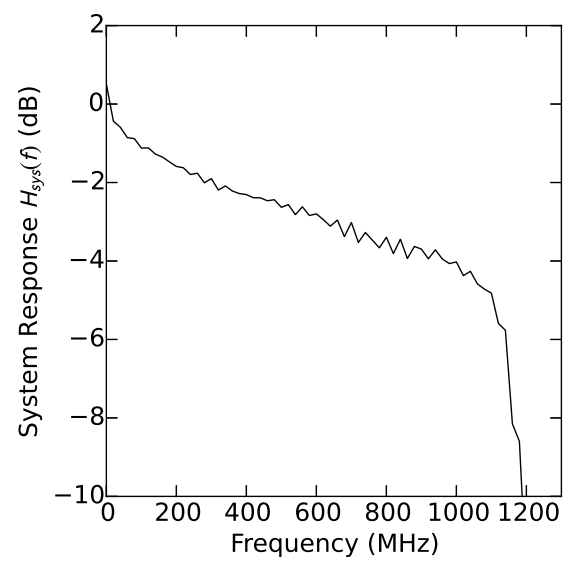

(b)

Figure 4: (a) The horizontally polarized effective height, $H_{e f f}(f)$, for several elevation angles relative to the boresight of the antennas. (b) A single channel system response, $H_{\text {sys }}(f)$, which includes the cables and filters used in the T-510 experiment.

The modulations in the power spectral density in both polarizations arise from the interference of reflections with the main pulse. This effect is evident in all of the measured power spectra, but is particularly prominent far from the center of the cone. The deep dips in the power spectra recored at $11.29 \mathrm{~m}$ and $1.30 \mathrm{~m}$ are due to the secondary reflection. The disagreement is strongest at low frequencies.

Adding in a reflection equivalent in amplitude to the initial pulse and at the time expected from geometry varies the simulated peak time-domain amplitude by a factor of 1.38 for the horizontally polarized signal at $6.52 \mathrm{~m}$. This indicates an uncertainty of $38 \%$ on the agreement between the data and the models, averaged over the band 300-1200 MHz. We exclude the 200-300 MHz band in the data and simulation comparisons due to uncertainty in the antenna response at low frequencies. The time-domain peaks of the data and simulations at this height differ by $36 \%$ and from the ZHS simulations by $34 \%$, commensurate with the systematic uncertainty.

The simulated and measured spectra are steeper at the top and bottom of the vertical scan, i.e., away from the peak of the cone, than at the center. This is expected due to frequency-dependent coherence effects and consistent with measurements from ANITA, in which the energy of a cosmic ray has been estimated from the total power in the RF spectrum $[9,10]$. The energy is degenerate with the angle between the measured radio emission and the Cherenkov angle. However, such a degeneracy can be broken by measuring the slope of the power spectrum, assuming that RF emission is conically-shaped and peaks at the Cherenkov angle. The cone-shaped emission is evident in the total power integrated in the 300-1200 MHz bands shown in Fig. 6.

\section{Conclusions}

We presented the first laboratory measurements of radio-frequency radiation from particle showers under the influence of a strong magnetic field, up to $\pm 970 \mathrm{G}$. Throughout the course of the run, the beam charge was $131 \pm 3.3 \mathrm{pC}$ and the beam energies were 4.33 and $4.55 \mathrm{GeV}$. Emission 

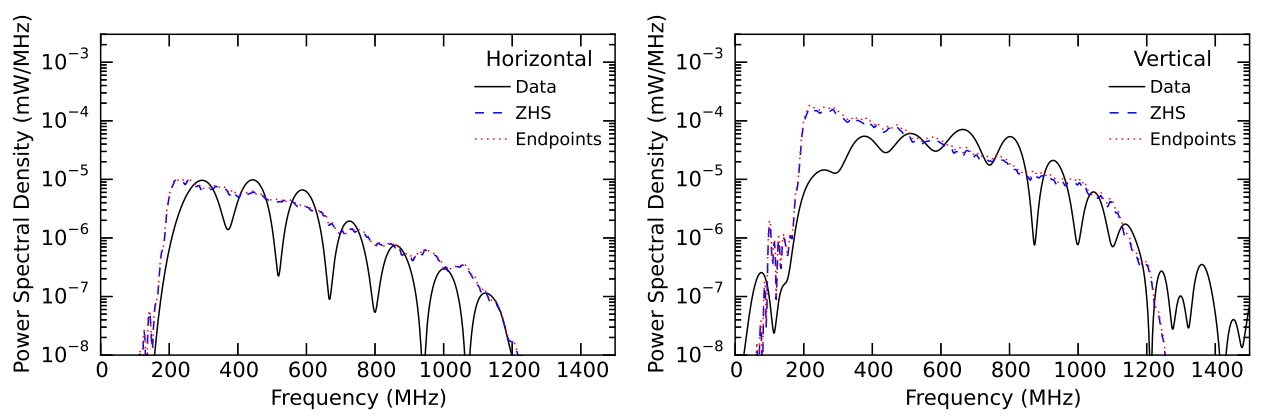

(a) $11.29 \mathrm{~m}$
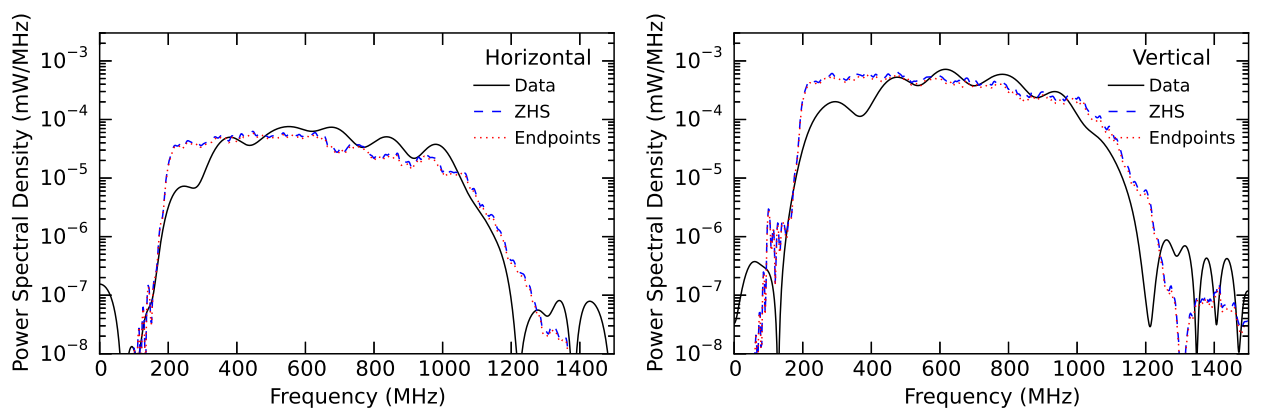

(b) $6.52 \mathrm{~m}$
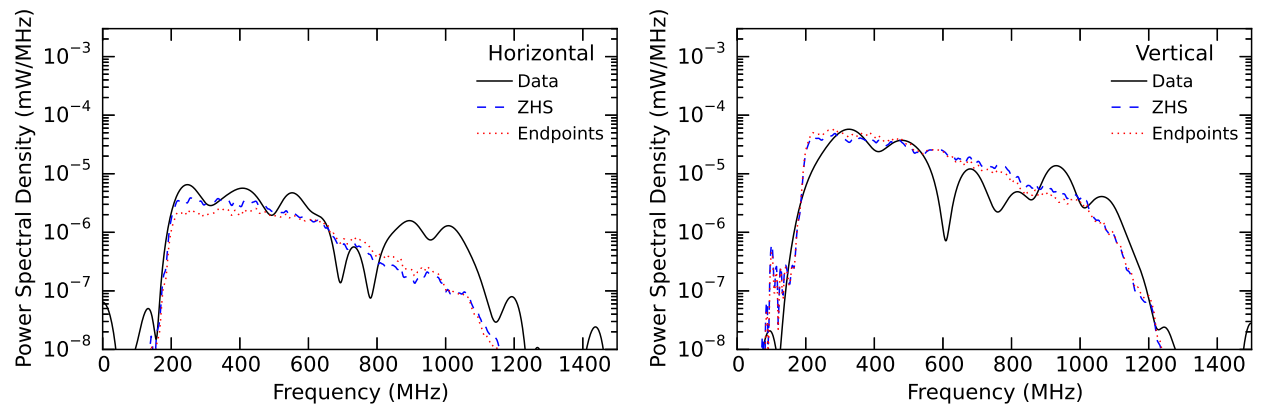

(c) $1.30 \mathrm{~m}$

Figure 5: Spectra for horizontally polarized emission (left) and vertically polarized emission (right) at full magnetic field strength compared with simulations from ZHS and Endpoints at three different heights.

induced by the magnetic field increases with field strength and forms a conical beam centered around the Cherenkov angle. The vertically polarized emission remains constant with respect to the magnetic field, as expected for Askaryan emission. The SLAC T-510 experiment validated the newly-developed first-principles based ZHS and Endpoints formalisms for radio emission from particle cascades, which have been shown to reproduce the data to within a systematic uncertainty of $38 \%$.

\section{Acknowledgements}

The authors thank SLAC National Accelerator Center for providing facilities and support and especially Janice Nelson and Carl Hudspeth for their support and dedication that made T-510 pos- 


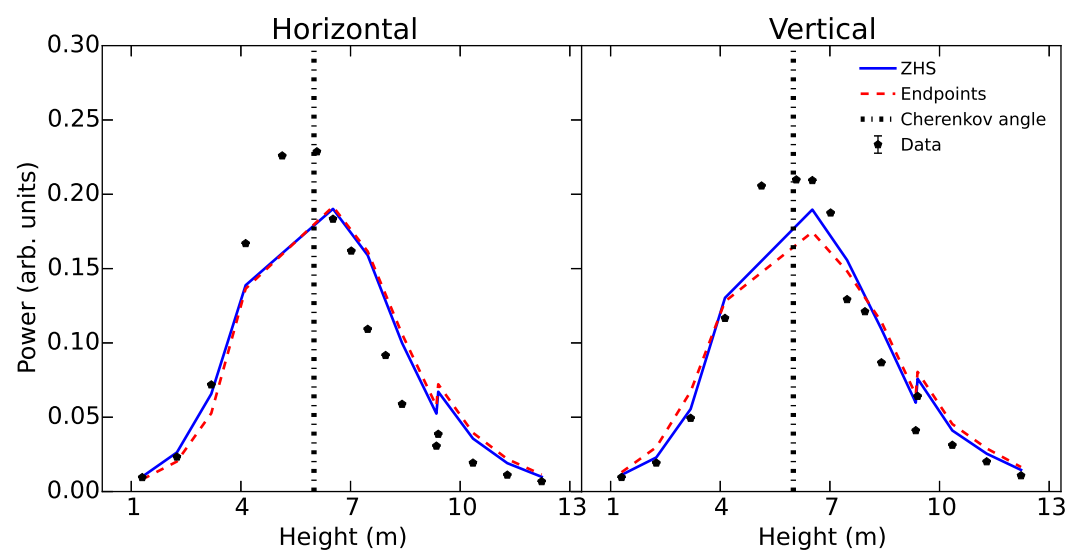

Figure 6: Integrated power in the 300-1200 MHz band throughout the vertical scan.

sible. Work supported in part by Department of Energy grants DE-AC02-76SF00515, the Taiwan Ministry of Science and Technology under project number MOST103-2119-M-002-002, and others. Part of this research was carried out at the Jet Propulsion Laboratory, California Institute of Technology, under a contract with the National Aeronautics and Space Administration.

\section{References}

[1] J. Alvarez-Muñiz, W. R. Carvalho Jr., and E. Zas, Monte Carlo simulations of radio pulses in atmospheric showers using ZHAireS, Astropart. Phys. 35 (2012), 6.

[2] T. Huege, M. Ludwig, and C. W. James. Simulating radio emission from air showers with CoREAS, in AIP Conf. Proc. 1535 (2013) 128 [astroph. HE/1301.2132].

[3] G. A. Askaryan, Excess Negative Charge of the Electron-Photon Shower and Coherent Radiation Originating from It, JETP 14 (1962) 441.

[4] G. A. Askaryan, Coherent Radio Emission from Cosmic Showers in Air and in Dense Media, JETP 21 (1965) 658.

[5] P. W. Gorham, et al., The Antarctic Impulsive Transient Antenna ultra-high energy neutrino detector: Design, performance, and sensitivity for the 2006-2007 balloon flight Astropart. Phys. 32 (2009), 10.

[6] K. Belov, et al., SLAC T-510: A beam-line experiment of radio emission from particle cascades in the presence of a magnetic field, Proc. 34th ICRC 2015 CR11 Radio \#346.

[7] A. Zilles, et al., Modeling of radio emission in the SLAC T-510 Experiment using microscopic Geant4 simulations, Proc. 34th ICRC 2015 CR-EX \#313.

[8] J. D. Krauss, Antennas MacGraw-Hill, 1988.

[9] K. Belov, et al. Towards determining the energy of the UHECRs observed by the ANITA detector, AIP Conf. Proc. 1535 (2013) 209.

[10] H. Schoorlemmer, et al., Energy and Flux Measurements of Ultra-High Energy Cosmic Rays Observed During the First ANITA Flight, submitted AstroPart. Phys, (2015)

[astroph. HE/1506.05396]. 